\title{
Comparison of etoricoxib and indomethacin for the treatment of experimental periodontitis in rats
}

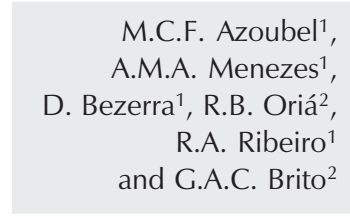

${ }^{1}$ Departamento de Fisiologia e Farmacologia,

${ }^{2}$ Departamento de Morfologia, Faculdade de Medicina,

Universidade Federal do Ceará, Fortaleza, CE, Brasil

\begin{abstract}
Correspondence
G.A.C. Brito

Departamento de Fisiologia

e Farmacologia

Faculdade de Medicina, UFC

Rua Coronel Nunes de Melo, 1127

60430-270 Fortaleza, CE

Brasil

Fax: +55-85-3366-8333

E-mail: gerlybrito@hotmail.com

Research supported by CNPq and Fundação Cearense de Pesquisa e Cultura (FUNCAP).
\end{abstract}

Received April 17, 2006 Accepted October 27, 2006

\begin{abstract}
We investigated the effect of etoricoxib, a selective cyclooxygenase2 inhibitor, and indomethacin, a non-selective cyclooxygenase inhibitor, on experimental periodontitis, and compared their gastrointestinal side effects. A ligature was placed around the second upper left molars of female Wistar rats (160 to $200 \mathrm{~g}$ ). Animals (6 per group) were treated daily with oral doses of 3 or $9 \mathrm{mg} / \mathrm{kg}$ etoricoxib, $5 \mathrm{mg} / \mathrm{kg}$ indomethacin, or $0.2 \mathrm{~mL}$ saline, starting 5 days after the induction of periodontitis, when bone resorption was detected, until the sacrifice on the 11th day. The weight and survival rate were monitored. Alveolar bone loss (ABL) was measured as the sum of distances between the cusp tips and the alveolar bone. The gastric mucosa was examined macroscopically and the periodontium and gastric and intestinal mucosa were examined by histopathology. The ongoing ABL was significantly inhibited $(\mathrm{P}<0.05)$ by 3 and $9 \mathrm{mg} / \mathrm{kg}$ etoricoxib and by indomethacin: control $=4.08 \pm 0.47 \mathrm{~mm}$; etoricoxib $(3 \mathrm{mg} / \mathrm{kg})$ $=1.89 \pm 0.26 \mathrm{~mm}$; etoricoxib $(9 \mathrm{mg} / \mathrm{kg})=1.02 \pm 0.14 \mathrm{~mm}$; indomethacin $=0.64 \pm 0.15 \mathrm{~mm}$. Histopathology of periodontium showed that etoricoxib and indomethacin reduced inflammatory cell infiltration, ABL, and cementum and collagen fiber destruction. Macroscopic and histopathological analysis of gastric and intestinal mucosa demonstrated that etoricoxib induces less damage than indomethacin. Animals that received indomethacin presented weight loss starting on the 7th day, and higher mortality rate (58.3\%) compared to etoricoxib (0\%). Treatment with etoricoxib, even starting when ABL is detected, reduces inflammation and cementum and bone resorption, with fewer gastrointestinal side effects.
\end{abstract}

\section{Introduction}

Periodontitis is a chronic inflammatory disease characterized by destruction of the tooth-supporting connective tissue and cementum, bone resorption, leukocyte infiltration, and periodontal pocket formation $(1,2)$.
Key words

- Alveolar bone loss

- Inflammation

- Periodontitis

- Cyclooxygenase inhibitors

- Etoricoxib

- Indomethacin

.....................
Although bacteria are considered to be the primary etiologic agents of periodontal disease $(3,4)$, the pathogenesis of periodontitis involves host-bacterial interaction followed by release of inflammatory mediators such as eicosanoids and cytokines (5). Arachidonic acid metabolites, mainly prostaglan- 
dins of the E series $\left(\mathrm{PGE}_{2}\right)$, seem to be critical mediators in the progression of periodontal disease. There is a large body of evidence suggesting an association of $\mathrm{PGE}_{2}$ levels within the periodontal tissues and within the crevicular fluid with the expression of clinical manifestations and bone destruction due to periodontal disease $(6,7)$.

PGs are synthesized from cell membrane phospholipids by action of cyclooxygenase (COX) enzymes (6,7). Three isoforms of COX have been recognized thus far (8). COX-1, the constitutive isoform, is found in almost all cells and has a role in normal physiological functions such as gastric cytoprotection and vascular and renal homeostasis. In contrast, COX-2 is expressed during the inflammatory reaction and mediates cell differentiation, mitogenesis and specialized signal transduction $(7,9,10)$. A third isoform of cyclooxygenase, named COX-3, has been recently described as a variant of $\mathrm{COX}-1$ which is most abundant in cerebral cortex and heart (8).

It has been suggested that the therapeutic activities of nonsteroidal anti-inflammatory drugs (NSAIDs) are derived from COX-2 blockade, whereas unwanted side effects such as gastrointestinal ulceration and bleeding, renal damage and platelet dysfunction are due to inhibition of COX-1 $(11,12)$. Thus, a selective blockade of the COX-2 isoform should lead to the inhibition of inflammation and pain without causing the COX-1-dependent side effects in gastric tissue and kidney $(13,14)$. However, it is important to emphasize that COX-2 inhibitors are contraindicated for patients with or at high risk of ischemic heart disease or stroke (15).

Etoricoxib is believed to provide gastrointestinal safety compared with nonselective NSAIDs and is rapidly and completely absorbed following oral administration, with a rapid onset of action (16). For this reason, we evaluated the oral administration of etoricoxib in the present study using a model of periodontitis.

Various studies have reported that the preventive use of NSAIDs can modify the progression of experimental periodontitis in rats (17). A recent study has documented a preventive benefit of etoricoxib (18). However, the mode of treatment with etoricoxib, a selective COX-2 inhibitor, has been poorly studied in periodontal disease models.

In our experimental model, we have used a previously unexplored delayed initiation of treatment with etoricoxib 5 days after experimentally induced periodontal disease in order to mimic the conditions of normal dentistry practice. Therefore, the aim of the present study was to evaluate the effect of treatment with etoricoxib, a selective COX2 inhibitor, compared with indomethacin, a non-selective COX inhibitor, on the progression of experimental periodontal disease, and to correlate the outcome with the potential gastric and intestinal mucosa side effects of these drugs.

\section{Material and Methods}

\section{Animals}

Female Wistar rats (160-200 g) from our own animal facilities were housed in temperature-controlled rooms and received water and food ad libitum. All experiments were conducted in accordance with local guidelines on the welfare of experimental animals and with the approval of the Ethics Committee of Animal Research of the Federal University of Ceará.

\section{Induction of inflammatory periodontal disease}

A sterilized nylon (000) thread ligature was placed around the cervix of the second left upper molar of rats anesthetized with $10 \%$ chloral hydrate $(400 \mathrm{mg} / \mathrm{kg}$, intraperitoneally) (19). The ligature was knotted on the buccal side of the tooth, resulting in a subgingival position palatally and in supragingival position buccally. The contralateral right side was used as the unligated control 
(20). Animals were weighed and survival rate was monitored daily.

\section{Drug treatments}

Animals subjected to experimental periodontitis (see above) were divided into equal groups of 6 animals each to receive an oral dose of $5 \mathrm{mg} / \mathrm{kg}$ indomethacin or 3 or $9 \mathrm{mg} /$ $\mathrm{kg}$ etoricoxib, po, starting 5 days after the induction of periodontitis and daily until sacrifice on the 11th day. Etoricoxib (Merck Sharp and Dohme, Withehouse Station, NJ, USA) was diluted in $2 \%$ DMSO (w/v) and sterile saline solution. Indomethacin (Merck Sharp and Dohme) was diluted in 5\% sodium bicarbonate $(\mathrm{w} / \mathrm{v})$ in sterile saline solution. The indomethacin $(5 \mathrm{mg} / \mathrm{kg})$ and etoricoxib (3 or $9 \mathrm{mg} / \mathrm{kg}$ ) doses were chosen as described elsewhere (21).

The results of these groups were compared to those for animals subjected to periodontitis that received $0.1 \mathrm{~mL} / 40 \mathrm{~g}$ saline, po (experimental periodontal disease (EPD) group; $\mathrm{N}=12$ ) and also to those for a group of 6 rats that received no manipulation (naive group). The same procedure was repeated in order to obtain maxillas for histopathology.

\section{Measurement of alveolar bone loss}

The animals were sacrificed on the 11th day after the induction of periodontitis with an overdose of ether. The maxillae were excised and fixed in $10 \%$ neutral formalin. Both maxillary halves were then defleshed and stained with $1 \%$ aqueous methylene blue in order to differentiate bone from teeth. The bone loss was analyzed using a stereoscope loupe (4X magnification) to measure the distance between the cusp tip and the alveolar bone, based on the method of Crawford et al. (22), modified (20). Measurements were made along the axis of each root of the first (three roots), second and third molar teeth (two roots). The total alveolar bone loss was obtained by taking the sum of the recordings from the buccal tooth surface and subtracting the value of the right maxilla (unligated control) from the left, in millimeters. Although it is claimed that laboratory rats may harbor ongoing baseline periodontitis (23), in the present study we used the contralateral right maxilla as the unligated control in order to avoid the influence of a baseline periodontitis on our results.

\section{Histopathological analysis}

The excised maxillae were fixed in $10 \%$ $(\mathrm{v} / \mathrm{v})$ buffered formalin and demineralized in a $7 \%(\mathrm{v} / \mathrm{v})$ nitric acid, followed by dehydration and paraffin embedding. The specimens were stained either with hematoxylineosin or Mallory trichrome which stain blue the collagen fibers of alveolar bone, cementum, and periodontal ligament. Six-micrometer thick sections obtained from 6 animals in each experimental group, corresponding to the area between the first and second molars where the ligature had been placed, were examined by light microscopy. A 0 to 3 scale was used for analysis: 0 , absence of or only mild cell infiltration (the inflammatory cell infiltration is sparse and restricted to the region of the marginal gingiva), preserved alveolar process and cementum; 1, moderate cell infiltration (the inflammatory cell infiltration is present over the entire inserted gingiva), minor alveolar process resorption and intact cementum; 2, severe cell infiltration (the inflammatory cell infiltration is present in both the gingivae and the periodontal ligament), extensive degradation of the alveolar process, and partial destruction of cementum; 3, severe cell infiltration, total destruction of the alveolar process and severe destruction of the cementum (24).

\section{Analysis of stomach and intestinal mucosa}

The stomachs and small intestines of the animals were removed, opened and macro- 
scopically analyzed for the presence of hyperemia (scores 0-1), loss of mucosal folds (scores 0-1), petechiae (scores 0-3), hemorrhage (scores 0-3), and ulcers (scores 0-3), according to previously described criteria (25). The organs were then fixed in $10 \%$ neutral formalin and embedded in paraffin. Five-micrometer thick sections were stained with $\mathrm{HE}$ and evaluated by light microscopy. The analysis of gastric lesions used a 0-3 scoring system: 0, absence of lesion; 1 , superficial erosion - damage to the surface epithelium only; 2 , deep erosion - glandular epithelium still present; 3 , presence of ulceration - as deep as the mucosal muscular layer (26). The analysis of the small intestine utilized a 0-3 scoring system: 0 , absence of lesion; 1 , mild lesion; 2 , moderate lesion; 3 , severe lesion, as proposed by Bertrand and colleagues (27).

\section{Statistical analysis}

Data are reported as means \pm SEM or as medians, when appropriate. Univariate ANOVA followed by the Bonferroni test was used to compare means, and the Kruskal-Wallis test was used to compare medians. $\mathrm{P}<0.05$ was taken to indicate significant differences.

\section{Results}

\section{Effect of etoricoxib treatment on experimental periodontal disease in rats}

Figure 1. Effect of etoricoxib and indomethacin on the alveolar bone loss in rats with experimental periodontal disease (EPD). Etoricoxib (Etori, 3 or $9 \mathrm{mg} / \mathrm{kg}$ ), indomethacin (Indo, $5 \mathrm{mg} / \mathrm{kg}$ ) or $0.2 \mathrm{~mL} 0.9 \%$ saline (EPD) was administered on the 5th day after the induction of periodontitis and daily until the 11th day, when the animals were sacrificed. Data are reported as the mean \pm SEM for at least 6 rats per group. ${ }^{*} \mathrm{P}<0.05$ compared to the EPD group (ANOVA followed by the Bonferroni test). tal disease caused a significant $(\mathrm{P}<0.05)$ and dose-dependent 53.53 and $74.87 \%$ inhibition of alveolar bone loss, respectively, as compared to the saline-treated rats. The response to $9 \mathrm{mg} / \mathrm{kg}$ etoricoxib was similar to that obtained with $5 \mathrm{mg} / \mathrm{kg}$ indomethacin $(84.22 \%)$ (Figure 1). There was no statistical difference between the two doses of etoricoxib or between 3 or $9 \mathrm{mg} / \mathrm{kg}$ etoricoxib and indomethacin. The naive group presented alveolar bone loss close to zero, i.e., there was a non-significant difference between the two sides of the maxilla. The macroscopic aspect of the periodontium from animals subjected to EPD for 11 days showed severe bone resorption with root exposure (Figure 2A), while the macroscopic feature of the periodontium from rats subjected to EPD and treated with $9 \mathrm{mg} / \mathrm{kg}$ etoricoxib, similar to $5 \mathrm{mg} / \mathrm{kg}$ indomethacin, showed considerable preservation of bone loss (Figure 2B). Histopathological analysis of the region between the first and second molars of the periodontium of animals subjected to experimental periodontitis (EPD group) demonstrated inflammatory cell infiltration, destruction of alveolar bone, collagen fiber derangement within the periodontal ligament, and resorption of cementum (Figure 2C), receiving a median score and range of 3 (2$3)$. The periodontium of rats treated with 9 $\mathrm{mg} / \mathrm{kg}$ etoricoxib (Figure 2D) or $5 \mathrm{mg} / \mathrm{kg}$ indomethacin showed preservation of the alveolar process and cementum, reduction of the inflammatory cell infiltration and partial preservation of collagen fibers of the periodontal ligament, receiving median score and range of 0 (0-1) and 1 (1-1), respectively. These values were statistically different $(\mathrm{P}<0.05)$ when compared to the EPD group.

All rats subjected to periodontitis lost weight as early as the first day of the experimental challenge. Etoricoxib-treated animals recovered weight similarly to the EPD rats treated with saline. Animals treated with indomethacin initially recovered the lost of weight, but suffered a rapid weight loss start- 
A

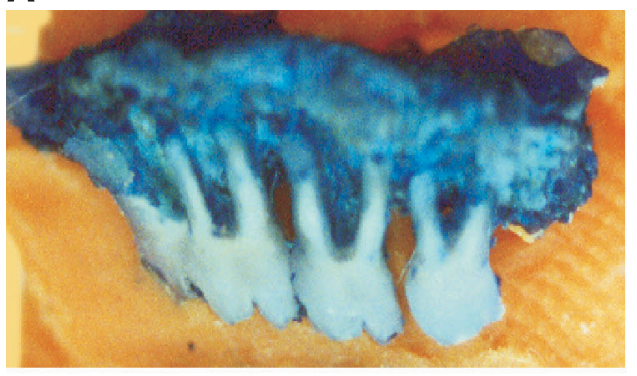

C

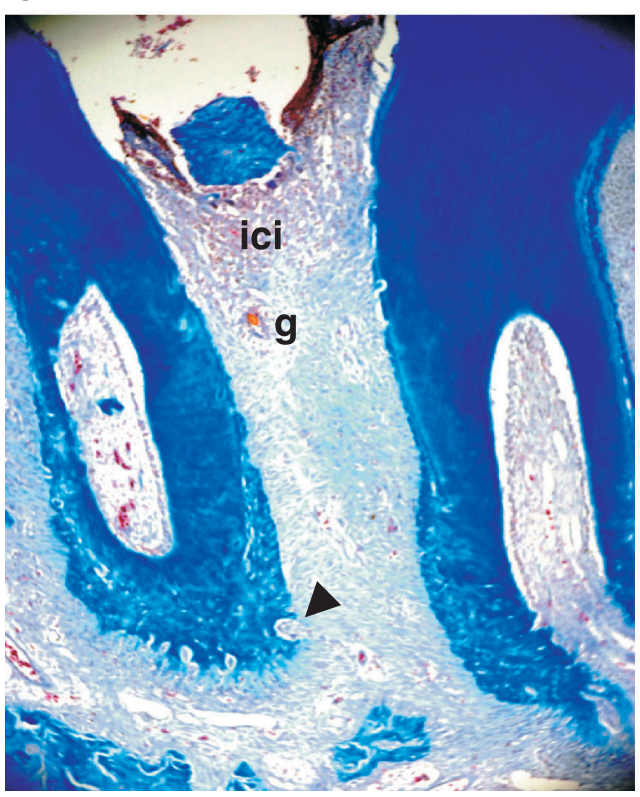

ing on the 7 th day ( 2 days after the beginning of treatment), which became significant after the 10th day following the ligature placement compared to the EPD group treated with saline (Figure 3). The survival curve, depicted in Figure 4, showed that all animals submitted to EPD and treated with etoricoxib survived until the end of the experiment, in contrast to the $58.3 \%$ mortality rate observed for the group treated with indomethacin. The survival rate was significantly lower in the indomethacin group compared to the etoricoxib group $(P=0.01$, chi-square test $)$.

\section{Effect of etoricoxib on gastric and intestinal mucosa damage}

Macroscopic analysis of the gastric mu-

\section{B}

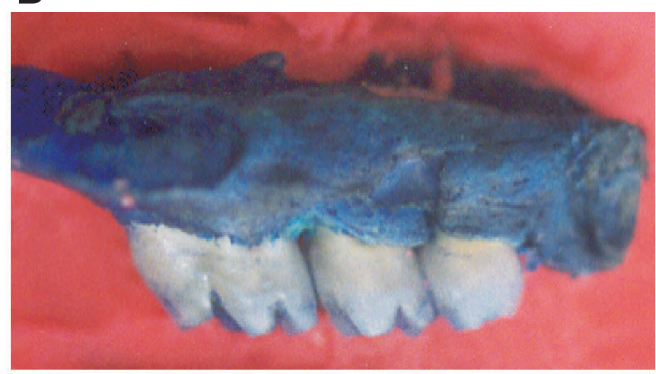

D
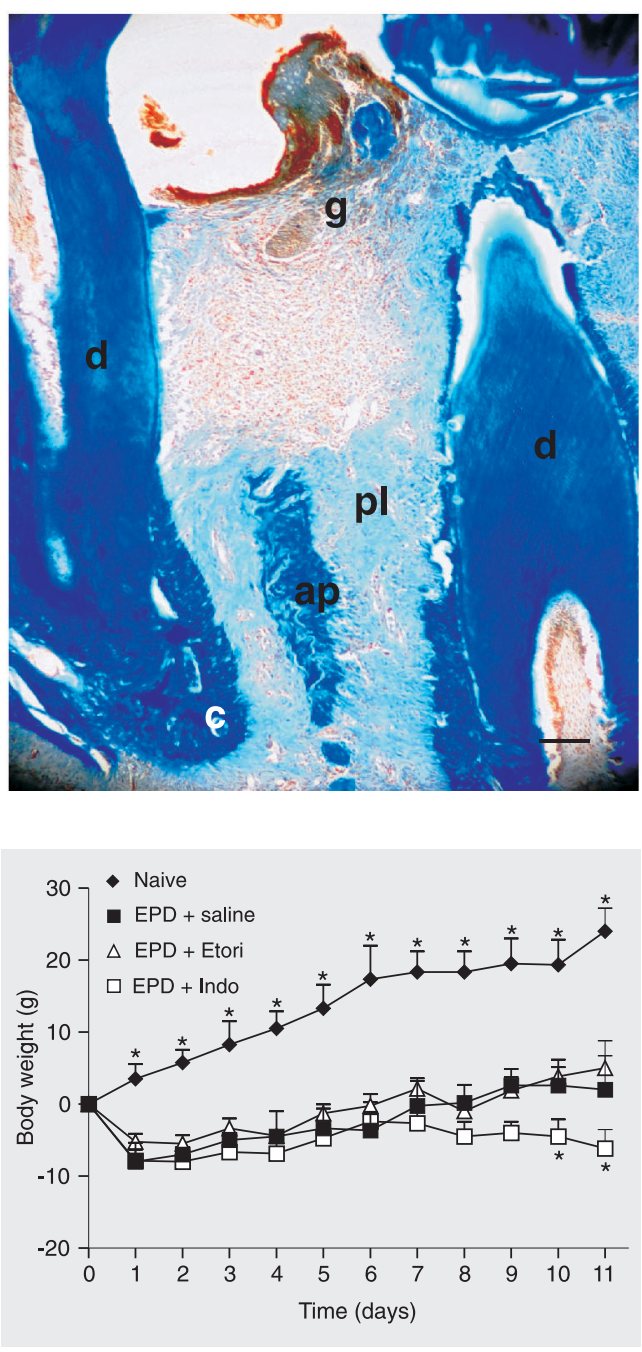

Figure 3. Effect of etoricoxib (Etori) and indomethacin (Indo) on body weight during experimental periodontal disease (EPD). Data are reported as the mean \pm SEM for 6 animals in each group. The experimental conditions are given in the legend to Figure 1, except that only $9 \mathrm{mg} / \mathrm{kg}$ etoricoxib was used. ${ }^{*} \mathrm{P}<0.05$ compared to EPD group (ANOVA followed by the Bonferroni test). The naive group of animals was not submitted to EPD.
Figure 2. Macroscopic aspects and histopathology of the periodontium of rats. A, Representative macroscopic aspect of the periodontium from an animal subjected to experimental periodontal disease (EPD) for 11 days, showing severe bone resorption with tooth root exposure; $B$, representative macroscopic feature of the periodontium from a rat subjected to EPD and treated with $9 \mathrm{mg} / \mathrm{kg}$ etoricoxib, showing considerable reduction of bone loss. Photomicrographs showing the region between the first and second molars of rats: $C$, representative histology of the periodontium from a rat subjected to EPD and treated with saline, showing inflammatory cell infiltration (ici) in the gingiva (g), cementum resorption (arrowhead) and total destruction of the alveolar process; $D$, periodontium of a rat subjected to EPD and treated with $9 \mathrm{mg} / \mathrm{kg}$ etoricoxib, showing preserved intraradicular periodontal ligament ( $\mathrm{pl}$ ) fibers, cementum (c), dentin (d), and alveolar process (ap). Mallory trichrome staining (original magnification: 100X; bar $=250 \mu \mathrm{m}$ ). 
cosa from the animals treated with $5 \mathrm{mg} / \mathrm{kg}$ indomethacin revealed loss of mucous membrane folds, hyperemia, petechiae, and hemorrhagic lesions receiving median score and range of 2 (1-4). These pathological changes were statistically significant $(\mathrm{P}<0.05)$ compared to the animals treated with $9 \mathrm{mg} / \mathrm{kg}$ etoricoxib and to the EPD group, which had no detectable macroscopic lesions in the stomach, except for the loss of mucosal folds in a few animals, receiving median scores and range of $1(0-1)$ for the etoricoxib group and $0.5(0-1)$ for the EPD group.

Histological analysis showed that the gastric mucosa of animals treated with $9 \mathrm{mg} /$ $\mathrm{kg}$ etoricoxib was similar to that of the animals not submitted to EPD (naive), receiving median scores and range of $0(0-1)$ for the etoricoxib group and $0(0-0)$ for the naive group, and comparable to that of the rats that only received saline solution (EPD group; 0 $(0-0)$ ), although we identified a superficial gastric erosion in only 1 animal of the etoricoxib group. In contrast, animals receiving indomethacin exhibited deep erosion of the glandular epithelium and a remarkable inflammatory cell infiltration, receiving median score and range of 2 (1-2).

Histopathological evaluation of the intestinal mucosa in the group treated with 9 $\mathrm{mg} / \mathrm{kg}$ etoricoxib demonstrated blunted villi in $50 \%$ of the rats. No histological alteration was seen in the naive or EPD groups. Villus necrosis and increased inflammatory cell infiltration were observed in the animals that

Figure 4. Effect of etoricoxib (Etori) and indomethacin (Indo) on the mortaliy of animals subjected to experimental periodontal disease (EPD) for 11 days. The survival curve was elaborated from the daily observation of animal deaths. The survival rate of the Indo group was significantly lower than that of the Etori group ( ${ }^{*} P=0.01$, chisquare test).

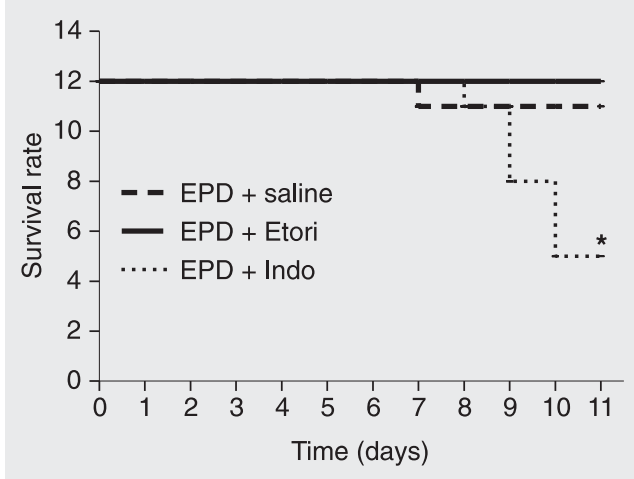

survived the indomethacin treatment, receiving median score and range of 2 (2-2) compared to the etoricoxib group $0.5(0-1)$ and the EPD group 0 (0-0). The indomethacintreated rats, which did not tolerate the experimental protocol, dying during the challenge, had increased peritoneal fluid and peritoneal fibrous adhesion (data not shown), suggesting peritonitis secondary to gastrointestinal perforation.

\section{Discussion}

We demonstrated the effect of orally active etoricoxib, a novel and highly selective COX-2 inhibitor $(28,29)$, on the progressive alveolar bone loss and the inflammatory response in response to thread ligature-induced periodontitis in adult rats. We also showed the advantage of etoricoxib for the integrity of the gastrointestinal mucosa as compared to indomethacin, a non-selective COX inhibitor. We used a delayed treatment model starting 5 days after the induction of periodontitis, when bone loss is already demonstrable (30). This approach more closely resembles the situation in clinical outpatient practice rather than an early treatment (18).

The role of eicosanoids released by COX2 activity in the response of the host to plaque-resident bacteria and mechanical distress during periodontitis has been well described $(4,30)$. PGs are believed to mediate the extensive connective tissue destruction and bone resorption occurring during symptomatic late periodontal disease (31). In this regard, it is reasonable to expect that $\mathrm{COX}$ 2 inhibition could potently reduce the negative outcome of periodontal disease. Thus, the intervention with orally administered etoricoxib on ongoing periodontal disease successfully reduced the edema, the collagen fiber degradation, the late mononuclear cell infiltration, and the cementum and bone resorption compared to the saline-treated controls, with efficacy similar to that of indomethacin intervention, although without 
the gastrointestinal side effects obtained with indomethacin.

The treatment of rats with a high dose (5 $\mathrm{mg} / \mathrm{kg}$ ) of the non-selective COX inhibitor, indomethacin, as compared to our previous study, which used $2 \mathrm{mg} / \mathrm{kg}$ indomethacin as preventive treatment (17), also showed a potent action, reducing the ongoing alveolar bone resorption (84\%). This effect is probably related to blockade of PG production and its action on bone remodeling (32), and to the reduction of the overall inflammatory process associated with experimental periodontitis. However, the inhibitory effect of indomethacin on PG production provoked severe gastrointestinal disturbances which could be related to poor weight recovery and higher mortality rates among the challenged rats. These findings are consistent with a large body of evidence relating non-selective COX inhibitors to gastrointestinal side effects obtained in both clinical trials and experimental studies (27,33-35).

Non-selective COX blockers probably cause ulcerogenic injury, not only by inhibiting COX-1, but also by inhibiting COX-2, since a growing body of evidence supports a beneficial role of constitutive COX-2 in the integrity of the gastrointestinal tract (36). Thus, it should be emphasized that selective COX-2 inhibitors might amplify prior gastrointestinal lesions induced by indomethacin, most likely by decelerating mucosal repair, a critical role recently claimed for COX-2 pathways in angiogenesis and tissue healing $(37,38)$. Therefore, these compounds are contraindicated for risk patients with a history of gastrointestinal disruption.

In our study, we found blunted villi in $50 \%$ of the small intestines of rats treated with etoricoxib. This mild alteration could be related to decelerating mucosal repair by COX-2 inhibition as reported by others (37, 38). However, most studies have demonstrated the gastrointestinal safety of systemic etoricoxib compared to non-selective COX inhibitors, a property usually shared by other COX-2-targeted inhibitors (39).

It is also important to emphasize the recently reported weak evidence of an increased cardiovascular risk with long-term use of etoricoxib in clinical trials (40). With the model used in the present study, it is not likely to obtain cardiovascular side effects because of the short duration of treatment with this COX-2 inhibitor. The use of highly selective COX-2 inhibitors should be contraindicated for patients at risk of cardiovascular diseases, especially the elderly (15).

From the viewpoint of public health, adequate oral hygiene is still the most important way to prevent periodontal disease and to control the progression of the disease. Once chronic periodontitis is clinically established, the careful use of selective COX2 inhibitors might constitute an important therapeutic tool for dental practice. Yet the short- and long-term effects of etoricoxib on human periodontal disease have been poorly examined. In view of the recent findings in COX-2 research, new studies are warranted to understand the role of these drugs, within the NSAID family, in the physiopathology of chronic periodontal disease and during long-term periodontal healing.

Using a highly reproducible experimental model of periodontitis in rats, we observed that the etoricoxib, a highly selective COX-2 inhibitor, is as effective as indomethacin in reducing inflammatory infiltration, collagen fiber destruction, cementum and bone resorption, although with fewer gastrointestinal side effects. Therefore, we suggest that etoricoxib may provide a better risk/benefit ratio in treatment of periodontitis than non-selective COX inhibitors.

\section{Acknowledgments}

The authors would like to thank José Ivan Rodrigues de Sousa and Maria Sivandira Pinheiro for skilled technical assistance. 


\section{References}

1. Williams RC. Periodontal disease. N Engl J Med 1990; 322: 373382.

2. Williams RC, Paquette DW. Understanding the pathogenesis of periodontitis: a century of discovery. J Int Acad Periodontol 2000; 2: 59-63.

3. Ximenez-Fyvie LA, Haffajee AD, Socransky SS. Comparison of the microbiota of supra- and subgingival plaque in health and periodontitis. J Clin Periodontol 2000; 27: 648-657.

4. Ximenez-Fyvie LA, Haffajee AD, Socransky SS. Microbial composition of supra- and subgingival plaque in subjects with adult periodontitis. J Clin Periodontol 2000; 27: 722-732.

5. Page RC. The etiology and pathogenesis of periodontitis. Compend Contin Educ Dent 2002; 23: 11-14.

6. Offenbacher S, Heasman PA, Collins JG. Modulation of host PGE2 secretion as a determinant of periodontal disease expression. $J$ Periodontol 1993; 64: 432-444.

7. Paquette DW, Williams RC. Modulation of host inflammatory mediators as a treatment strategy for periodontal diseases. Periodontology 2000; 24: 239-252.

8. Chandrasekharan NV, Dai H, Roos KL, Evanson NK, Tomsik J, Elton TS, et al. COX-3, a cyclooxygenase-1 variant inhibited by acetaminophen and other analgesic/antipyretic drugs: cloning, structure, and expression. Proc Natl Acad Sci USA 2002; 99: 1392613931.

9. Jouzeau JY, Terlain B, Abid A, Nedelec E, Netter P. Cyclo-oxygenase isoenzymes. How recent findings affect thinking about nonsteroidal anti-inflammatory drugs. Drugs 1997; 53: 563-582.

10. Steinmeyer J. Pharmacological basis for the therapy of pain and inflammation with nonsteroidal anti-inflammatory drugs. Arthritis Res 2000; 2: 379-385.

11. Vane JR, Botting RM. Anti-inflammatory drugs and their mechanism of action. Inflamm Res 1998; 47 (Suppl 2): S78-S87.

12. Lohinai Z, Stachlewitz R, Szekely AD, Feher E, Dezsi L, Szabo C. Evidence for the expression of cyclooxygenase-2 enzyme in periodontitis. Life Sci 2001; 70: 279-290.

13. Engelhardt G, Homma D, Schlegel K, Utzmann R, Schnitzler C. Anti-inflammatory, analgesic, antipyretic and related properties of meloxicam, a new non-steroidal anti-inflammatory agent with favourable gastrointestinal tolerance. Inflamm Res 1995; 44: 423433.

14. Engelhardt G. Pharmacology of meloxicam, a new non-steroidal anti-inflammatory drug with an improved safety profile through preferential inhibition of COX-2. Br J Rheumatol 1996; 35 (Suppl 1): 412.

15. Savage R. Cyclo-oxygenase-2 inhibitors: when should they be used in the elderly? Drugs Aging 2005; 22: 185-200.

16. Matsumoto AK, Cavanaugh PF Jr. Etoricoxib. Drugs Today 2004; 40: 395-414.

17. Bezerra MM, de Lima V, Alencar VB, Vieira IB, Brito GA, Ribeiro RA, et al. Selective cyclooxygenase-2 inhibition prevents alveolar bone loss in experimental periodontitis in rats. J Periodontol 2000; 71: 1009-1014.

18. Holzhausen M, Spolidorio DM, Muscara MN, Hebling J, Spolidorio LC. Protective effects of etoricoxib, a selective inhibitor of cyclooxygenase-2, in experimental periodontitis in rats. $J$ Periodontal Res 2005; 40: 208-211.

19. Sallay K, Sanavi F, Ring I, Pham P, Behling UH, Nowotny A. Alveolar bone destruction in the immunosuppressed rat. $J$ Periodontal
Res 1982; 17: 263-274.

20. Samejima Y, Ebisu S, Okada H. Effect of infection with Eikenella corrodens on the progression of ligature-induced periodontitis in rats. J Periodontal Res 1990; 25: 308-315.

21. Banerjee AK, Peters TJ. Experimental non-steroidal anti-inflammatory drug-induced enteropathy in the rat: similarities to inflammatory bowel disease and effect of thromboxane synthetase inhibitors. Gut 1990; 31: 1358-1364.

22. Crawford JM, Taubman MA, Smith DJ. The natural history of periodontal bone loss in germfree and gnotobiotic rats infected with periodontopathic microorganisms. J Periodontal Res 1978; 13: 316325.

23. Bjornsson MJ, Velschow S, Stoltze K, Havemose-Poulsen A, Schou $S$, Holmstrup P. The influence of diet consistence, drinking water and bedding on periodontal disease in Sprague-Dawley rats. $J$ Periodontal Res 2003; 38: 543-550.

24. Leitao RF, Ribeiro RA, Chaves HV, Rocha FA, Lima V, Brito GA. Nitric oxide synthase inhibition prevents alveolar bone resorption in experimental periodontitis in rats. J Periodontol 2005; 76: 956-963.

25. Szabo S, Trier JS, Brown A, Schnoor J, Homan HD, Bradford JC. A quantitative method for assessing the extent of experimental gastric erosions and ulcers. J Pharmacol Methods 1985; 13: 59-66.

26. Trevethick MA, Clayton NM, Strong $P$, Harman IW. Do infiltrating neutrophils contribute to the pathogenesis of indomethacin induced ulceration of the rat gastric antrum? Gut 1993; 34: 156-160.

27. Bertrand V, Guimbaud R, Tulliez M, Mauprivez C, Sogni P, Couturier $D$, et al. Increase in tumor necrosis factor-alpha production linked to the toxicity of indomethacin for the rat small intestine. $\mathrm{Br} \mathrm{J}$ Pharmacol 1998; 124: 1385-1394.

28. Riendeau D, Percival MD, Brideau C, Charleson S, Dube D, Ethier $D$, et al. Etoricoxib (MK-0663): preclinical profile and comparison with other agents that selectively inhibit cyclooxygenase-2. J Pharmacol Exp Ther 2001; 296: 558-566.

29. Martina SD, Vesta KS, Ripley TL. Etoricoxib: a highly selective COX-2 inhibitor. Ann Pharmacother 2005; 39: 854-862.

30. Zubery Y, Dunstan CR, Story BM, Kesavalu L, Ebersole JL, Holt SC, et al. Bone resorption caused by three periodontal pathogens in vivo in mice is mediated in part by prostaglandin. Infect Immun 1998; 66: 4158-4162.

31. Miyauchi M, Hiraoka M, Oka H, Sato S, Kudo Y, Ogawa I, et al. Immuno-localization of COX-1 and COX-2 in the rat molar periodontal tissue after topical application of lipopolysaccharide. Arch Oral Biol 2004; 49: 739-746.

32. Carter-Bartlett P, Dersot JM, Saffar JL. Periodontal and femoral bone status in periodontitis-affected hamsters receiving a high dose indomethacin treatment. J Biol Buccale 1989; 17: 93-101.

33. Nygard G, Anthony A, Piasecki C, Trevethick MA, Hudson M, Dhillon $A P$, et al. Acute indomethacin-induced jejunal injury in the rat: early morphological and biochemical changes. Gastroenterology 1994; 106: 567-575.

34. Lugea A, Antolin M, Mourelle M, Guarner F, Malagelada JR. Deranged hydrophobic barrier of the rat gastroduodenal mucosa after parenteral nonsteroidal anti-inflammatory drugs. Gastroenterology 1997; 112: 1931-1939.

35. Chmaisse HM, Antoon JS, Kvietys PR, Grisham MB, Perry MA. Role of leukocytes in indomethacin-induced small bowel injury in the rat. Am J Physiol 1994; 266: G239-G246.

36. Tanaka A, Hase S, Miyazawa T, Takeuchi K. Up-regulation of cyclo- 
oxygenase-2 by inhibition of cyclooxygenase-1: a key to nonsteroidal anti-inflammatory drug-induced intestinal damage. J Pharmacol Exp Ther 2002; 300: 754-761.

37. Laudanno OM, Cesolari JA, Esnarriaga J, Rista L, Piombo G, Maglione $\mathrm{C}$, et al. Gastrointestinal damage induced by celecoxib and rofecoxib in rats. Dig Dis Sci 2001; 46: 779-784.

38. Gudis K, Sakamoto C. The role of cyclooxygenase in gastric mu- cosal protection. Dig Dis Sci 2005; 50 (Suppl 1): S16-S23.

39. Scheiman JM. Gastroduodenal safety of cyclooxygenase-2 inhibitors. Curr Pharm Des 2003; 9: 2197-2206.

40. Aldington S, Shirtcliffe P, Weatherall M, Beasley R. Systematic review and meta-analysis of the risk of major cardiovascular events with etoricoxib therapy. N Z Med J 2005; 118: U1684. 